\title{
Schwannoma de cauda equina com acometimento vertebral: relato de caso e revisão de literatura
}

\section{Cauda equina schwannoma with vertebral damage: case report and literature review}

Lucas Fernandes Ferreira ${ }^{1}$. Karen Lopes Cunha ${ }^{1}$. Marcos André Araújo Accioly Filho ${ }^{1}$. Rayanne Cristina Cavalcante Vieira ${ }^{1}$. Gabriel Albuquerque Parente ${ }^{1}$. Arnaldo Ribeiro de Arruda ${ }^{2}$. José Arnaldo Motta de Arruda ${ }^{3}$.

1 Academico(a) de Medicina da Universidade Federal do Ceará (UFC) e membro do Núcleo de Estudos em Neurocirurgia, Fortaleza, Ceará, Brasil. 2 Médico neurocirurgião. 3 Médico neurocirurgião, Doutorado em Medicina (Neurocirurgia), professor do Departamento de Cirurgia da Universidade Federal do Ceará (UFC), Fortaleza, Ceará, Brasil.

\section{RESUMO}

O schwannoma é uma neoplasia de células de Schwann a qual pode ocorrer em diversas partes do sistema nervoso, mas corresponde a uma pequena parcela dos tumores de cauda equina, sendo incomum o acometimento ósseo. Relatamos o caso de um paciente masculino, 49 anos, o qual apresentou quadro álgico com redução de força. Exames de imagem revelaram tumor na região da cauda equina causando compressão nervosa e erosão do corpo vertebral de L3. Os achados histopatológicos corroboram diagnóstico de schwannoma e o tratamento cirurgico acarreta resolução do quadro.

Palavras-chave: Coluna vertebral. Neoplasias. Neurocirurgia.

\section{ABSTRACT}

The schwannoma is a Schwann cell neoplasm that can occur in many regions of the nervous system, although it accounts for a small portion of the cauda equina tumors being uncommon the bone involvement. We report the case of a male patient, 49 years old, presenting algic condition with strength reduction. Imaging revealed a cauda equina tumor causing nerve compression and L3 vertebral body erosion. Histopathological findings support the schwannoma diagnosis and the surgical treatment causes solution of the condition.

Keywords: Spine. Neoplasms. Neurosurgery.

Autor correspondente: Lucas Fernandes Ferreira, Rua Desembargador Praxedes, 1455, Vila União, Fortaleza, Ceará. CEP: 60420-025.Telefone: +5585 98899-7986. E-mail: Iff_lucas@hotmail.com

Conflito de interesses: Não há qualquer conflito de interesses por parte de qualquer um dos autores.

Recebido em: 12 Fev 2016; Revisado em: 07 Jun 2016; Aceito em: 29 Jul 2016. 


\section{INTRODUÇÃO}

O schwannoma, também conhecido como neurilemoma, é uma neoplasia de células de Schwann, as quais são responsáveis pela formação da bainha de mielina de nervos periféricos. Tal tumor tem caráter não metastático na maioria dos pacientes e sua incidência é menor que 0,5 por 100.000 pessoas por ano. Sua prevalência aumenta com a idade, sendo o pico entre a quarta e a quinta décadas de vida. ${ }^{1}$

Essa neoplasia tem como sua localização mais comum o nervo vestíbulo-coclear (NC VIII), principalmente seu ramo vestibular, embora também possa ocorrer em outros nervos cranianos ou na medula espinal. Apenas 6\% dos tumores intradurais, categoria na qual se encaixam a maioria dos schwannomas, ocorrem no nível da cauda equina. ${ }^{2}$

Quando há a presença de múltiplos schwannomas, é possível que exista associação com neurofibromatose (tipo I ou tipo II) ou com schwannomatose, doenças de caráter hereditário. A primeira se manifesta com schwannoma do NC VIII, também conhecido como schwannoma do acústico. Segundo estudos de grande porte, a neurofibromatose, contudo, acomete apenas cerca de $7 \%$ dos pacientes com schwannoma, sendo mais comum que se encontre essa neoplasia sem doença associada. ${ }^{1}$ A presença de schwanoma bilateral do nervo acústico é patognomônico de Neurofibromatose tipo II.

O estudo histopatológico de um schwannoma clássico demonstra alternância entre áreas mais densas e áreas menos celulares.

As principais manifestações clínicas do schwannoma são tardias, uma vez que o tumor apresenta crescimento lento, e decorrem da compressão nervosa causada pela massa. Dentre elas estão dor e alterações na sensibilidade ou na motricidade. Em raras situações há acometimento ósseo associado.

\section{RELATO DO CASO}

Paciente do sexo masculino, 49 anos, apresentou lombocialtagia esquerda, acompanhada de redução aguda da força desse membro (grau IV), por um período de três anos de forma progressiva, quando da consulta o paciente apresentava nítida paresia do membro inferior esquerdo, predominando a atrofia do quadríceps femoral. Foi realizada ressonância nuclear magnética, a qual demonstrou uma massa tumoral causando grande erosão óssea no corpo vertebral de L3 (Figura 1). A remoção da neoplasia foi realizada por meio de uma hemilaminectomia com extirpação total do tumor com preservação da raiz nervosa de L3 e L4 à esquerda. Apesar de haver lesão óssea, não foi necessária a realização de fixação da coluna, uma vez que as facetas articulares e o disco intervertebral não foram comprometidos, preservando-se a estabilidade.

O paciente apresentou importante melhora no quadro álgico da região lombar e da perna esquerda após o procedimento. A biópsia do tumor confirmou o diagnóstico de schwannoma, inclusive com visualização das áreas de Antoni A e Antoni B. Não houve recidivas do tumor durante o acompanhamento. Três anos após a cirurgia, o paciente voltou às suas atividades de trabalho e a atrofia do músculo quadríceps femoral regrediu completamente.

Figura 1. Ressonância Nuclear Magnética evidenciando schwannoma erodindo corpo vertebral.

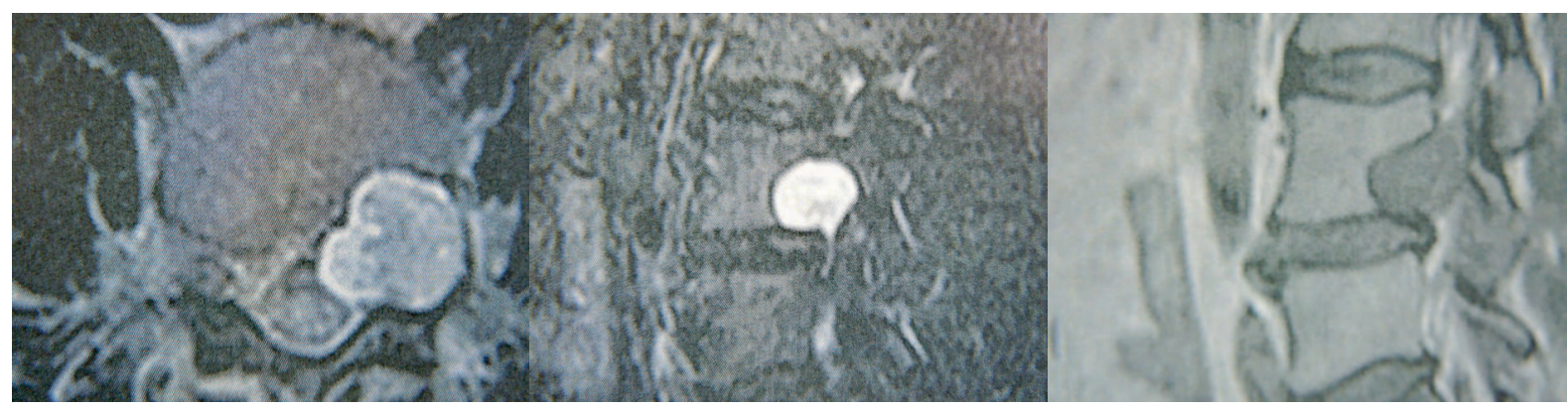

\section{DISCUSSÃO}

Dentre todos os tumores espinais, $86,6 \%$ não têm caráter metastático e apenas $0,5 \%$ ocorrem no nível da cauda equina. ${ }^{3}$ Essas neoplasias são classificadas como extradurais, intradurais extramedulares ou intradurais intramedulares. O schwannoma é um tumor de bainha nervosa, sendo, portanto, intradural extramedular, embora alguns possam crescer a ponto de terem componente extradural ou mesmo serem completamente extradurais, ${ }^{1,4}$ havendo inclusive relato de casos em que o schwannoma não se liga ao tecido nervoso. ${ }^{5}$ Em geral, o schwannoma de cauda equina clássico apresenta crescimento lento, o que torna seus sintomas tardios. Quando sintomático, esse tumor causa, na maioria dos casos, dor, a qual piora durante a noite, e alterações na sensibilidade, uma vez que a neoplasia normalmente se estabelece em raízes sensitivas. Schwannomas gigantes podem ainda causar distúrbios do sistema nervoso autônomo, como incontinência urinária. ${ }^{6}$ No caso relatado, a neoplasia se encontrava no nível da cauda equina e tinha origem intradural, embora se estendesse além dos limites do canal vertebral. Tal expansão gerou erosão óssea vertebral por pressão, causando remodelamento do osso. $\mathrm{O}$ acometimento ósseo pode ser presente em schwannomas em outras localizações, ${ }^{7}$ sendo muito comum em schwanomas no nível do sacro. ${ }^{8}$ A incidência de erosão vertebral em neurinomas de cauda equina, como o visto no caso relatado, não é bem elucidada em grandes estudos, 
embora raros casos de extensa erosão óssea por neoplasia nessa localização já tenham sido descritos. ${ }^{5}$

Esse tumor também pode ter uma apresentação plexiforme com uma forma multinodular, embora essa variante seja mais comum em região de cabeça e pescoço, sendo extremamente rara na região da cauda equina tendo apenas um caso sido relatado na literatura de schwannoma plexiforme em cauda equina. ${ }^{9}$

Clinicamente, é possível que a mielopatia ou radiculopatia, como no caso relatado, decorrente de uma compressão nervosa causada por schwannoma, seja confundida com herniação de disco, tumor ou mesmo, mais raramente, doenças desmielinizantes.

O diagnóstico primário do schwannoma é realizado por técnicas de imagem, ao se avaliar, por exemplo, o formato do tumor, sendo o schwannoma mais arredondado e o neurofibroma, seu principal diagnóstico diferencial, de formato mais fusiforme. Contudo, tais técnicas não são sempre precisas na diferenciação entre esse tumor e um neurofibroma. Além disso, outros tumores intradurais extramedulares também podem ter apresentação similar à do schwannoma, como o meningioma. Para o diagnóstico do schwannoma o método mais sensível e mais específico é a ressonância nuclear magnética. Em geral, o tumor se apresenta como uma massa de intensidade de sinal moderada em T1 e de intensidade de sinal alta em T2. Na maioria dos casos, a neoplasia é visualizada contida ao espaço do canal medular, comprimindo o nervo. Nesse caso houve clara erosão óssea da vértebra L3, decorrente do crescimento tumoral. Entretanto, as facetas articulares foram poupadas, o que evitou a necessidade de fixação da coluna.

\section{REFERÊNCIAS}

1. Conti P, Pansini G, Mouchaty H, Capuano C, Conti R. Spinal neurinomas: retrospective analysis and long-term outcome of 179 consecutively operated cases and review of the literature. Surg Neurol. 2004;61(1):34-44.

2. Wostrack M, Shiban E, Obermueller T, Gempt J, Meyer B, Ringel F. Conus medullaris and cauda equina tumors: clinical presentation, prognosis, and outcome after surgical treatment: clinical article. J Neurosurg Spine. 2014;20(3):335-43.

3. Jung KW, Park KH, Ha J, Lee SH, Won YJ, Yoo H. Incidence of primary spinal cord, spinal meninges, and cauda equina tumors in Korea, 2006-2010. Cancer Res Treat. 2015;47(2):166-72.

4. Celli P, Trillò G, Ferrante L. Spinal extradural schwannoma. J Neurosurg Spine. 2005;2(4):447-56.

5. Zhang F, Lu F, Jiang J, Wang H. Two case reports and an updated review of spinal intraosseous schwannoma. J Korean Neurosurg Soc. 2015;57(6):478-83.

6. Iizuka Y, Iizuka H, Kobayashi R, Mieda T, Takagishi K. Giant schwannoma with extensive scalloping of the lumbar vertebral body
O método de tratamento utilizado, laminectomia com extirpação total do tumor, é o mais utilizado para o tratamento dessa neoplasia, embora certas vezes seja realizada ressecção subtotal. ${ }^{10} \mathrm{~A}$ ressecção subtotal, contudo, pode estar relacionada a altas taxas de reincidências, havendo estudos que indicaram existir recorrência de todos os tumores submetidos a ressecção parcial. ${ }^{1}$ A ressecção subtotal pode ainda, em certos casos, negligenciar tumores com potencial maligno. ${ }^{11}$ Tais evidências corroboram a decisão de extirpação total da neoplasia no caso relatado, assim como a ausência de recidivas durante o acompanhamento.

Além disso, há controvérsias quanto à incidência de instabilidade vertebral após a laminectomia, embora tal complicação não tenha acontecido com o paciente cujo caso é relatado.

A confirmação diagnóstica de um schwannoma se dá pelo estudo histopatológico, o qual classicamente demonstra alternância de camadas compactas de células fusiformes, paliçadas alternadas com os corpos de Verocay, denominadas áreas de Antoni $\mathrm{A}$, e camadas menos celulares e mais desorganizadas que podem apresentar vacúolos lipídicos, denominadas áreas de Antoni B.

Dessa forma, é importante que se tenha em mente o fato de o neurinoma de cauda equina, apesar de ser um tumor de lento crescimento, ter potencial para causar grandes erosões vertebrais. A possibilidade de incidência de instabilidade vertebral também não deve ser esquecida nesses casos ao se optar pelo tratamento cirúrgico com laminectomia, devendo a possibilidade de ser feita fixação da coluna ser avaliada em cada caso.

treated with one-stage posterior surgery: a case report. J Med Case Rep. 2014;8:421.

7. Baranović M, Macan D, Begović EA, Luksic I, Brajdić D, Manojlović S. Schwannoma with secondary erosion of mandible: case report with a review of the literature. Dentomaxillofac Radiol. 2006;35(6):456-60.

8. Salvant JB Jr, Young HF. Giant intrasacral schwannoma: an unusual cause of lumbrosacral radiculopathy. Surg Neurol. 1994;41(5):411-3.

9. Mori K, Imai S, Nishizawa K, Nakamura A, Ishida M, Matsusue Y. Plexiform schwannoma arising from cauda equina. Spine J. 2015;15(1):205-6.

10. Sohn S, Chung CK, Park SH, Kim ES, Kim KJ, Kim CH. The fate of spinal schwannomas following subtotal resection: a retrospective multicenter study by the Korea spinal oncology research group. J Neurooncol. 2013;114(3):345-51.

11. Bhatia S, Khosla A, Dhir R, Bhatia R, Banerji AK. Giant lumbosacral nerve sheath tumors. Surg Neurol. 1992;37(2):118-22.

\section{Como citar:}

Ferreira LF, Cunha KL, Accioly MA Filho, Vieira RC, Parente GA, Arruda AR, et al. Schwannoma de cauda equina com acometimento vertebral: relato de caso e revisão de literatura. Rev Med UFC. 2017 jan-abr;57(1):56-58. 